\title{
Mortality surveillance in the Netherlands: severity of winter 2016/2017
}

\author{
Liselotte van Asten ${ }^{\star 1}$, Marit de Lange ${ }^{1}$, Anne Teirlinck ${ }^{1}$, Lenny Stoeldraijer ${ }^{2}$, \\ Carel Harmsen ${ }^{2}$ and Wim van der Hoek ${ }^{1}$
}

${ }^{1}$ Centre for Infectious Disease Control Netherlands, National Institute for Public Health and the Environment (RIVM), Utrecht, Netherlands; ${ }^{2}$ Statistics Netherlands, The Hague, Netherlands

\begin{abstract}
Objective
Weekly numbers of deaths are monitored to increase the capacity to deal with both expected and unusual (disease) events such as pandemic influenza, other infections and non-infectious incidents. The monitoring information can potentially be used to detect, track and estimate the impact of an outbreak or incident on all-cause mortality.
\end{abstract}

\section{Introduction}

The mortality monitoring system (initiated in 2009 during the influenza $\mathrm{A}(\mathrm{H} 1 \mathrm{~N} 1)$ pandemic) is a collaboration between the Centre for Infectious Disease Control (CIb) of National Institute for Public Health and the Environment (RIVM) and Statistics Netherlands. The system monitors nation-wide reported number of deaths (population size 2017: 17 million) from all causes, as cause of death information is not available real-time. Data is received from Statistics Netherlands by weekly emails.

\section{Methods}

Once a week the number of reported deaths is checked for excess above expected levels at 2 different time-lags: within 1 and 2 weeks after date of death (covering a median $43 \%$ and $96 \%$ of all deaths respectively). A weekly email bulletin reporting the findings is sent to the Infectious Disease Early Warning Unit (at $\mathrm{CIb}$ ) and a summary of results is posted on the RIVM website. Any known concurrent and possibly related events are also reported. When excess deaths coincide with hot temperatures, the bulletin is sent to the Heat Plan Team (also at RIVM). Data are also sent to EuroMOMO which monitors excess mortality at a European level. For the Dutch system baselines and prediction limits are calculated using a 5 year historical period (updated each July). A serfling-like algorithm based on regression analysis is used to produce baselines which includes cyclical seasonal trends (models based on historical data in which weeks with extreme underreporting have been removed. Also periods with high excess mortality in winter and summer were removed so as not to influence the baseline with previous outbreaks).

\section{Results}

Increased mortality started two weeks after the influenza epidemic started and remained increased during the remainder of the influenza epidemic except for a drop in week 52 (coinciding with Christmas holidays) (Figure 1). Excess mortality was primarily observed in persons 75 years or older. Cumulative excess mortality was estimated at 7,503 deaths occurring during the 15 weeks of the 2016/2017 influenza epidemic (week 48 of 2016 through week 10 of 2017) and at 8,890 during the total winter season (44 weeks running from week 40 up to week 20 of the next year).

\section{Conclusions}

In terms of number of deaths during the winter season (weeks 40-20 the next year) and during the influenza epidemic (weeks 4810 ), the $2016 / 2017$ season in the Netherlands was of high severity compared with the previous five seasons. Mortality was only higher in the $2014 / 2015$ season when the longest influenza epidemic was recorded of 21 weeks.

\section{Keywords}

Mortality; surveillance; influenza

\section{*Liselotte van Asten}

E-mail: liselotte.van.asten@rivm.nl 